\title{
TEM characterization of novel nano-hybrid materials
}

\author{
M. Saunders, ${ }^{*}$ S. Iyer, ${ }^{* *}$ C. Raston, ${ }^{* *}$ C. Bond ${ }^{* * *}$ \\ ${ }^{1}$ Centre for Microscopy, Characterisation and Analysis, The University of Western Australia, \\ Crawley, Perth, WA 6009, Australia \\ ${ }^{2}$ Centre for Strategic Nanofabrication, The University of Western Australia, Crawley, Perth, WA \\ 6009, Australia \\ ${ }^{3}$ School of Biomedical, Biomoleular and Chemical Sciences, The University of Western Australia, \\ Crawley, Perth, WA 6009, Australia
}

Fabricating nanostructures with well-defined shape and size is of considerable interest because of their unique chemical and physical properties. It is well known that simultaneous fine control of the size and shape of nanomaterials in building up novel structures is rather challenging. Developing techniques and synthetic protocols to gain access to new nanostructures of higher complexity is important in defining and fine-tuning the shape- and size-dependent properties of nanomaterials for end use applications. Transmission electron microscopy (TEM) plays a critical role in this development process, providing essential information on the structure and composition of these materials at the nano- and atomic-scale.

Within the Centre for Strategic Nanofabrication at The University of Western Australia, research is being conducted into the templated growth and self-assembly of novel nano-hybrid materials. For example, Iyer, et al. have developed a method for the hierarchical aqueous self-assembly of $\mathrm{C}_{60}$ nano-whiskers and $\mathrm{C}_{60}$-silver nano-hybrids under continuous flow [1]. In this approach, a starchiodine complex is used as a template to self-assemble $\mathrm{C}_{60}$ molecules into nano-whiskers, which are subsequently coated in silver (Fig. 1). The resulting materials have been characterized via a combination of high resolution imaging and diffraction [1].

Another approach uses duplex DNA as a template to grow silver triangles and nanoplates [2]. In this case (Fig. 2), a combination of TEM imaging, electron diffraction and energy-dispersive x-ray microanalysis is being used to investigate the structural and compositional properties of the resulting nano-hybrid materials.

\section{References}

[1] K.S. Iyer, et al., Lab Chip 7, 1121-1124 (2007).

[2] K.S. Iyer, et al., Crystal Growth \& Design 8(5), 1451-1453 (2008).

[3] The authors acknowledge the facilities, scientific and technical assistance of the Australian Microscopy \& Microanalysis Research Facility at the Centre for Microscopy, Characterisation \& Analysis, The University of Western Australia, a facility funded by The University, State and Commonwealth Governments. 

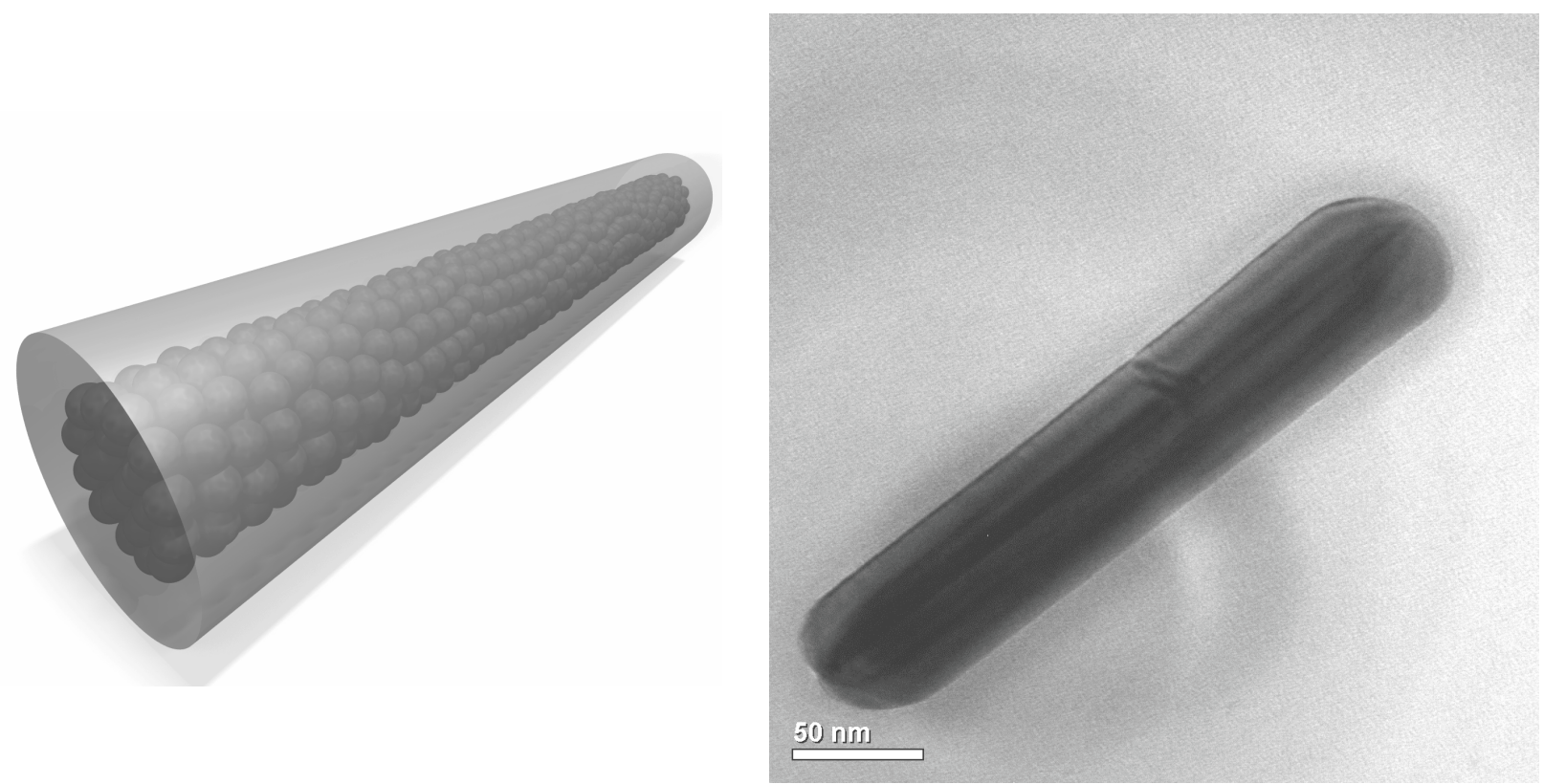

Fig. 1. Schematic (left) and TEM image (right) of a $\mathrm{C}_{60}$-silver nano-hybrid. High resolution imaging and electron diffraction have been used to confirm the core-shell configuration [1].
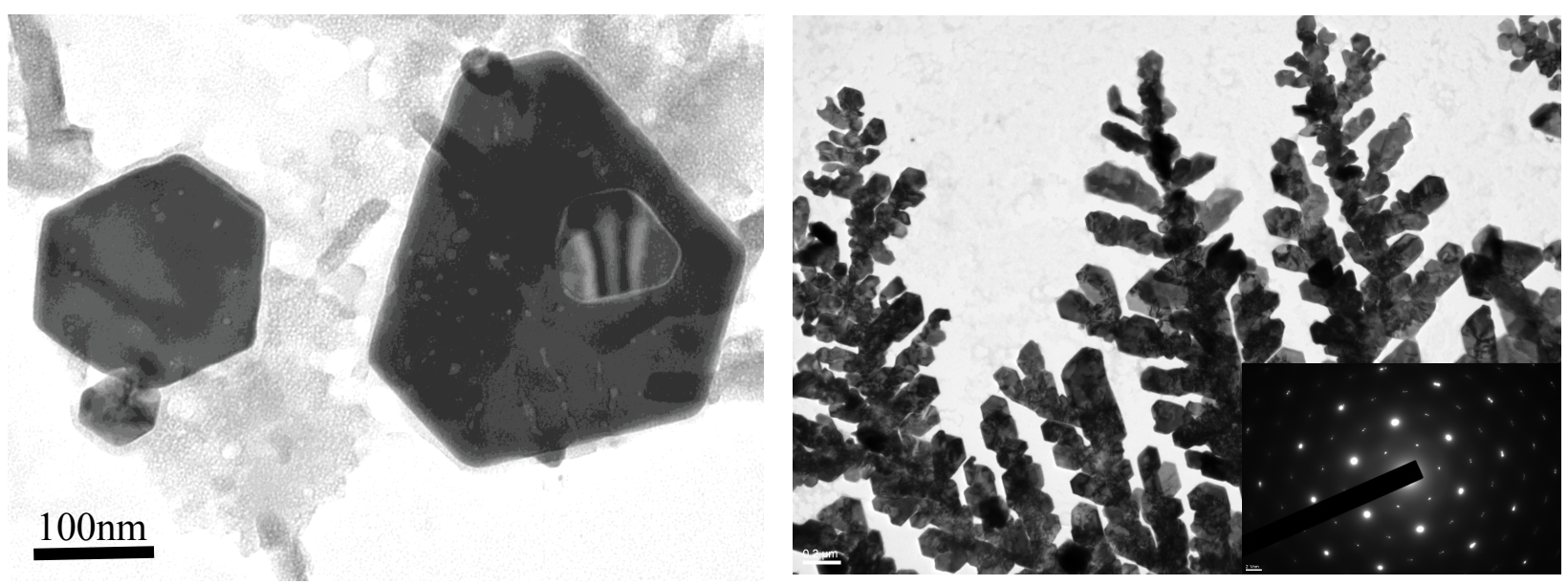

Fig 2. Using duplex DNA as a template, two silver nanostructure morphologies have been produced. The first (left) are flat hexagonal plates $\sim 5-10 \mathrm{~nm}$ thick bounded by a thin layer of DNA on their surface with concentric silver/DNA/silver/DNA nanoplate structures occasionally being formed. The second (right) are large, micron scale two-dimensional dendritic structures consisting of interconnecting silver plates, which have a thin layer of DNA on the outer surface. Selected area diffraction from the dendritic structures (inset) shows that the individual silver plates grow to form a large, single crystal network of silver. 